We report the results of a consensus conference on the diagnosis of multiple system atrophy (MSA). We describe the clinical features of the disease, which include four domains: autonomic failure/urinary dysfunction, parkinsonism and cerebellar ataxia, and corticospinal dysfunction. We set criteria to define the relative importance of these features. The diagnosis of possible MSA requires one criterion plus two features from separate domains. The diagnosis of probable MSA requires the criterion for autonomic failure/urinary dysfunction plus poor levodopa responsive parkinsonism or cerebellar ataxia. The diagnosis of definite MSA requires pathological confirmation.

Keywords: multiple system atrophy, parkinsonism, cerebellar ataxia, autonomic insufficiency, urinary dysfunction, glial cytoplasmic inclusions.

\section{Consensus statement on the diagnosis of multiple system atrophy}

\author{
Sid Gilman, Phillip Low, Niall Quinn, \\ Alberto Albanese, Yoav Ben-Shlomo, \\ Clare Fowler, Horacio Kaufmann, Thomas \\ Klockgether, Anthony Lang, Peter Lantos, \\ Irene Litvan, Christopher Mathias, Eugene \\ Oliver, David Robertson, Irwin Schatz, and \\ Gregor Wenning*
}

Address correspondence to Dr. Sid Gilman, Professor \& Chair, Department of Neurology, University of Michigan Medical Center, 1500 E. Medical Center Drive/1914 TC, Ann Arbor, MI 48109-0316, USA.

Tel: (734) 936-9070; Fax: (734) 763-5059

E-mail: sgilman@umich.edu

Received Mar 7, 1998; accepted July 13, 1998
Multiple system atrophy (MSA), a progressive neurodegenerative disease of undetermined etiology, occurs sporadically and causes parkinsonism and cerebellar, autonomic, urinary, and pyramidal dysfunction in many combinations [1-4]. The disease affects both sexes, usually beginning in middle age and progressing over intervals of 1 to 18 years, with a median survival of 9.3 years from the first symptom $[5,6]$. The parkinsonian features include bradykinesia with rigidity, postural instability, hypokinetic speech, and often tremor, usually with a poor or unsustained response to chronic levodopa therapy. The cerebellar dysfunction consists of ataxia of gait, limb movements and speech, and disorders of extraocular movements [7]. Autonomic insufficiency results in orthostatic hypotension, often with an inadequate heart rate response to standing, male erectile dysfunction (MED), constipation, and decreased sweating $[8,9]$. Urinary symptoms include urgency, frequency, nocturia, incomplete bladder emptying, and incontinence [10]. The diagnosis of MSA requires primarily clinical assessment; however, a number of laboratory tests may help to support the diagnosis.

The neuropathological changes consist of a high density of glial cytoplasmic inclusions (GCIs) in association with degenerative changes in some or all of the following structures: putamen, caudate nucleus, globus pallidus, substantia nigra, locus ceruleus, inferior olives, pontine nuclei, cerebellar Purkinje cells, autonomic nuclei of the brainstem, and the intermediolateral cell columns and Onufs nucleus in the spinal cord $[11,12]$. GCIs are ubiquitin-, tau- and a-synuclein-positive oligodendroglial inclusions [12].
Some efforts have been made to establish diagnostic criteria [4], but no consistent detailed guidelines have been developed. Accordingly, a consensus conference was convened on April 23 and 24, 1998 in Minneapolis, Minnesota, cosponsored by the American Autonomic Society and the American Academy of Neurology. The goal of the conference was to develop guidelines for the diagnosis of MSA. We achieved consensus on the items listed below and shown in Tables 1,2 , and 3. These guidelines have not yet been validared, and will almost certainly require further modification in the light of future experience.

\section{Clinical domains}

\section{Autonomic and urinary dysfunction}

Orthostatic hypotension $(\mathrm{OH})$ may indicate autonomic failure and can be asymptomatic or symptomatic. When symptomatic, it typically occurs after the onset of MED and urinary symptoms. Symptoms of $\mathrm{OH}$ result from cerebral hypoperfusion, and syncope may occur. The consensus conference determined that the clinical diagnosis of probable MSA requires a reduction of systolic blood pressure by at least $30 \mathrm{~mm} \mathrm{Hg}$ or of diastolic blood pressure by at least 15 $\mathrm{mm} \mathrm{Hg}$ within 3 minutes of standing from the recumbent

* Please see "Conference Participants" section at the end of this article for a full listing of author affiliations. 
Table 1. Clinical domains, features, and criteria used in the diagnosis of $\mathrm{MSA}^{*}$

I. Autonomatic and urinary dysfunction

A) Autonomic and urinary features

1. Orthostatic hypotension (by $20 \mathrm{~mm} \mathrm{Hg}$ systolic or 10 $\mathrm{mm} \mathrm{Hg}$ diastolic)

2. Urinary incontinence or incomplete bladder emptying

B) Criterion for autonomic failure or urinary dysfunction in MSA

Orthostatic fall in blood pressure (by $30 \mathrm{~mm} \mathrm{Hg}$ systolic or 15 $\mathrm{mm} \mathrm{Hg}$ diastolic) or urinary incontinence (persistent, involuntary partial or total bladder emptying, accompanied by erectile dysfunction in men) or both

II. Parkinsonism

A) Parkinsonian features

1. Bradykinesia (slowness of voluntary movement with progressive reduction in speed and amplitude during repetitive actions)

2. Rigidity

3. Postural instability (not caused by primary visual, vestibular, cerebellar, or proprioceptive dysfunction)

4. Tremor (postural, resting, or both)

B) Criterion for parkinsonism in MSA

Bradykinesia plus at least one of items 2 to 4

III. Cerebellar dysfunction

A) Cerebellar features

1. Gait ataxia (wide based stance with steps of irregular length and direction)

2. Ataxic dysarthria

3. Limb ataxia

4. Sustained gaze-evoked nystagmus

B) Criterion for cerebellar dysfunction in MSA

Gait ataxia plus at least one of items 2 to 4

IV. Corticospinal tract dysfunction

A) Corticospinal tract features

1. Extensor plantar responses with hyperreflexia

B) Corticospinal tract dysfunction in MSA: no corticospinal tract features are used in defining the diagnosis of MSA

${ }^{*} A$ feature $(A)$ is a characteristic of the disease and a criterion $(B)$ is a defining feature or composite of features required for diagnosis.

position. Frequently this is accompanied by an inadequate increase in heart rate (less than 10 beats per minute). We note that this is a more pronounced degree of $\mathrm{OH}$ than established previously [4]. MED appears early and affects virtually all male parients with MSA, but the symptom has

Table 2. Diagnostic categories of $\mathrm{MSA}^{*}$

I. Possible MSA: One criterion plus two features from separate domains. When the criterion is parkinsonism, a poor levodopa response qualifies as one feature (hence only one additional feature is required).

II. Probable MSA: Criterion for: autonomic failure/urinary dysfunction plus poor levodopa-responsive parkinsonism or cerebellar dysfunction.

III. Definite MSA: Pathologically confirmed by the presence of a high density of glial cytoplasmic inclusions in association with a combination of degenerative changes in the nigrostriatal and olivopontocerebellar pathways.

*The features and criteria for each clinical domain are shown in Table 1.
Table 3. Exclusion criteria for the diagnosis of MSA

I. History

Symptomatic onset under 30 years of age

Family history of a similar disorder

Systemic diseases or other identifiable causes for features listed in Table 1

Hallucinations unrelated to medication

II. Physical examination

DSM criteria for dementia

Prominent slowing of vertical saccades or vertical supranuclear gaze palsy*

Evidence of focal cortical dysfunction such as aphasia, alien limb syndrome, and parietal dysfunction

III. Laboratory Investigation

Metabolic, molecular genetic, and imaging evidence of an alternative cause of features listed in Table 1

*In practice, MSA is most frequently confused with Parkinson's disease or progressive supranuclear palsy (PSP) [25]. Mild limitation of upward gaze alone is nonspecific, whereas a prominent $(>50 \%)$ limitation of upward gaze or any limitation of downward gaze suggests PSP. Before the onset of vertical gaze limitation, a clinically obvious slowing of voluntary vertical saccades is usually easily detectable in PSP and assists in the early differentiation of these two disorders [26].

low specificity. Urinary frequency, urgency, incontinence, or incomplete bladder emptying also occur early and commonly.

\section{Parkinsonism}

The majority of MSA patients develop parkinsonian features at some stage of the disorder. All these patients have bradykinesia; rigidity, postural instability, and tremor also often occur. The tremor is usually irregular and postural, often incorporating myoclonus. A classical pill-rolling parkinsonian rest tremor is uncommon. The parkinsonism in MSA can be asymmetric. The dysarthria is mainly hypokinetic, often mixed with other components [13]. The parkinsonian features usually respond poorly to chronic levodopa therapy; however, up to $30 \%$ of patients show a clinically significant response to levodopa therapy at some time in the course, but the response is usually sustained for less than five years $[5,14,15]$. These are the most challenging patients for accurate diagnosis.

\section{Cerebellar dysfunction}

Ataxia of gait, the most common cerebellar feature of MSA, often occurs accompanied by dysarthria, limb ataxia, and sometimes gaze-evoked nystagmus and ocular dysmetria. A common finding is saccadic pursuit movements. The dysarthria in patients with predominantly cerebellar dysfunction is mainly ataxic, often mixed with other components [13].

\section{Corticospinal dysfunction}

Extensor plantar responses with hyperreflexia occur in about $50 \%$ of MSA patients. Corticospinal signs can contribute to the diagnosis, but are less important than abnormalities in the other domains. 


\section{Response to levodopa}

Levodopa responsiveness should be tested by administering escalating doses (with a peripheral decarboxylase inhibitor) over a 3-month period up to at least $1 \mathrm{~g}$ per day (if necessary and if tolerated). A positive response is defined as clinically significant improvement. This should be demonstrated by objective evidence such as an improvement of $30 \%$ or more on part III (motor examination) of the Unified Parkinson's Disease Rating Scale [16].

\section{Laboratory investigations}

Autonomic function tests, sphincter electromyography (EMG), and neuroimaging may be used to support the diagnosis, and neuroimaging is helpful in excluding other conditions. The abnormalities described below have been defined principally in clinically well-established cases rather than in the early stages of the disease. In the early stages, the tests may give equivocal results. We consider it premature to incorporate laboratory results into the entirely clinical guidelines that we established, but envision the future development of "Laboratory Supported" diagnostic categories.

Assessment of autonomic function can be assisted by a comprehensive battery that evaluates the distribution and severity of sudomotor, cardiovagal, and sympathetic adrenergic deficits $[17,18]$. Autonomic function tests may help separate MSA from Parkinson's disease and from idiopathic cerebellar degenerations [8].

Sphincter EMG can be useful in the diagnosis of MSA. Analysis of individual motor unit potentials recorded from the external anal sphincter usually shows changes indicating chronic reinnervation, with markedly prolonged motor units $[10,19]$.

Magnetic resonance imaging (MRI) can assist the evaluation by detecting abnormalities of striatum, cerebellum, and brainstem, but can be normal in up to $20 \%$ of cases [20]. Striatal abnormalities may include putaminal atrophy, slitlike signal change at the posterolateral putaminal margin, and hypointensity of the putamen relative to the globus pallidus [21]. Infratentorial abnormalities include cerebellar and pontine atrophy, and signal change in the pons and middle cerebellar peduncles [22]. Studies are in progress to evaluate the utility of magnetic resonance spectroscopy, positron emission tomography, and single photon emission tomography.

\section{Diagnostic categories}

We established three diagnostic categories reflecting differing levels of certainty: definite, probable, and possible. The diagnosis of definite MSA can only be made after neuropathological examination of the central nervous system revealing the characteristic density and distribution of GCIs and degenerative changes outlined above. The diagnosis of probable or possible MSA can be made using different combinations of clinical domains, criteria, and features, as indicated in Tables 1 and 2. Exclusion criteria are shown in Table 3.

\section{Terminology}

MSA is a distinct clinicopathological entity. The term should not be used to describe other neurodegenerative diseases affecting multiple systems. The use of confusing terms such as "multisystem degeneration" for MSA is inappropriate and now should be discouraged. We recommend designating patients as having MSA-P if parkinsonian features predominate or MSA-C if cerebellar features predominate $[23,24]$. These terms are intended to replace the striatonigral degeneration (SND) and sporadic olivopontocerebellar atrophy (sOPCA) types of MSA, respectively. The term Shy Drager syndrome has been widely misused, and is no longer useful.

\section{Acknowledgment}

This conference was supported in part by the Office of Rare Diseases and the National Institute of Neurological Disorders and Stroke, National Institutes of Health; Glaxo Wellcome, Inc.; Hoechst Marion Roussell; and Roberts Pharmaceutical Corporation.

\section{Conference participants}

Sid Gilman, M.D., Department of Neurology, University of Michigan Medical Center, Ann Arbor, MI

Phillip Low, M.D., Department of Neurology, Mayo Clinic, Rochester, MN

Niall Quinn, M.D., and Clare Fowler, M.D., Department of Neurology, National Hospital for Neurology and Neurosurgery, Queen Square, London, England

Alberto Albanese, M.D., Department of Neurology, Universita Cattolica, Istituto di Neurologia, Roma, Italy

Yoav Ben-Shlomo, M.D., Department of Social Medicine, Canynge Hall, Bristol, England

Horacio Kaufmann, M.D., Department of Neurology, Mt. Sinai Medical Center, New York, NY

Thomas Klockgether, M.D., Department of Neurology, University of Bonn, Bonn, Germany

Anthony Lang, M.D., Department of Neurology, Toronto Western Hospital, Toronto, Ontario, Canada

Peter Lantos, M.D., Ph.D., Department of Neuropathology, Institute of Psychiatry, London England

Irene Litvan, M.D., National Institutes of Health, Federal Building, Bethesda, MD

Christopher Mathias, D.Phil. D.Sc., Department of Neurovascular Medicine, Imperial College School of Medicine at St. Mary's, London, England, and Department of Neurology, National Hospital for Neurology and Neurosurgery, Queen Square, London, England

David Robertson, M.D., Departments of Pharmacology and Neurology, Vanderbilt University, Nashville, TN 
Irwin Schatz, M.D., Department of Medicine, University of Hawaii at Manoa, Honolulu, HI

Gregor Wenning, M.D., Universitaets-Klinik fur Neurologie, Innsbruck, Austria

\section{References}

1. Graham JG, Oppenheimer DR. Orthostatic hypotension and nicotine sensitivity in a case of multiple system atrophy. $J$ Neurol Neurosurg Psychiat 1969; 32:28-34.

2. Quinn N. Multiple system atrophy. In: Marsden CD, Fahn S, eds. Movement disorders. London: Butterworths, 1996; 262-281.

3. Albanese A, Colosimo C, Bentivoglio AR, et al. Multiple system atrophy presenting as parkinsonism: clinical features and diagnostic criteria. J Neurol Neurosurg Psychiat 1995; 59:144-151.

4. Consensus Committee of the American Autonomic Society and the American Academy of Neurology. Consensus statement on the definition of orthostatic hypotension, pure autonomic failure, and multiple system atrophy. Neurology 1996; 46:1470.

5. Wenning GK, Ben-Shlomo Y, Magalaes M, Daniel SE, Quinn NP. Clinical features and natural history of multiple system atrophy: an analysis of 100 cases. Brain 1994; 117:835-845.

6. Klockgether $T$, Lüdtke $R$, Kramer $B$, et al. The natural history of degenerative ataxia: a retrospective study in 466 patients. Brain 1998; 121:589-600.

7. Gilman S. Multiple System Atrophy. In: Jankovic J, Tolosa E, eds. Parkinson's disease and movement disorders, 3rd edition. Baltimore: Williams and Wilkins, 1998; 245-262.

8. Sandroni P, Ahlskog JE, Fealey RD, Low PA. Autonomic involvement in extrapyramidal and cerebellar disorders. Clin Auton Res 1991: 1:147-155.

9. Mathias CJ, Williams AC. The Shy Drager Syndrome (and Multiple System Atrophy). In: Neurodegenerative diseases. Calne DB, ed. Philadelphia: WB Saunders, 1994; 743-768.

10. Beck RO, Betts CD, Fowler CJ. Genitourinary dysfunction in multiple system atrophy: clinical features and treatment in 62 cases. J Urology 1994; 151:1336-1341.

11. Daniel SE. The neuropathology and neurochemistry of multiple system atrophy. In: Bannister R, Mathias CJ, eds. Autonomic failure: a textbook of clinical disorders of the autonomic nervous system. Oxford: Oxford University Press; 1992:564-585.
12. Lantos PL. Multiple system atrophy. Brain Pathology 1997; 7:1293-1297.

13. Kluin KJ, Gilman $S$, Lohman $M$, Junck L. Characteristics of the dysarthria of multiple system atrophy. Arch Neurol 1996; 53:545-548.

14. Hughes AJ, Colosimo C, Kleedorfer B, Daniel SE, Lees AJ. The dopaminergic response in multiple system atrophy. I Neurol Neurosurg Psychiatry 1992; 55:1009-1013.

15. Parati EA, Fetoni V, Geminiani GC, et al. Response to L-DOPA in multiple system atrophy. Clin Neuropharmacol 1997; 16:139-144.

16. Fahn S, Elton R. Unified Parkinson's Disease Rating Scale Committee: Unified Parkinson's disease rating scale. In: Fahn S, Marsden CD, Calne D, eds. Recent developments in Parkinson's disease. New York: Macmillan; 1987:153-164.

17. Mathias CJ, Bannister R. Investigation of Autonomic Disorders. In: Autonomic failure. A textbook of clinical disorders of the autonomic nervous system. 3rd edition. Bannister $\mathrm{R}$, Mathias $\mathrm{CJ}$, eds. Oxford: Oxford University Press; 1992:255-290.

18. Low PA. The composite autonomic scoring scale for the laboratory quantitation of generalized autonomic failure. Mayo Clin Proc 1993; 68:748-752.

19. Palace J, Chandiramani VA, Fowler CJ. Value of sphincter electromyography in the diagnosis of multiple system atrophy. Muscle Nerve 1997; 20:1396-1403.

20. Schrag A, Kingsley D, Phatouros $C$, et al. Clinical usefulness of magnetic resonance imaging in multiple system atrophy. $J$ Neurol Neurosurg Psychiatry 1998; 65:65-71.

21. Lang $A E$, Curran $T$, Provias J, Bergeron C. Striatonigral degeneration: iron deposition in putamen correlates with the slit-like void signal of magnetic resonance imaging. Can J Neurol Sci 1994; 21:311-318.

22. Testa D, Savoiardo M, Fetoni V, et al. Multiple system atrophy. Clinical and MR observations on 42 cases. Ital J Neuro Sci 1993; 14:211-216.

23. Mathias CJ. Autonomic dysfunction. Brit $J$ Hosp Med 1987; 38:238-243.

24. Schulz JB, Klockgether T, Petersen D, et al. Multiple system atrophy: natural history, MRI morphology, and dopamine receptor imaging with 123IBZM-SPECT. $J$ Neurol Neurosurg Psychiatry 1994; 57:1047-1056.

25. Litvan I, Goetz CG, Jankovic J, et al. What is the accuracy of the clinical diagnosis of multiple system atrophy? A clinicopathologic study. Arch Neurol 1997; 54:937-944.

26. Litvan I, Agid Y, Calne D, et al. NINDS-SPSP clinical criteria for the diagnosis of progressive supranuclear palsy (Steele-Richardson(Olszewski syndrome). Neurology 1996; 47:1-9. 